Chapter 7

\title{
Design and Application of Piezoelectric Stacks in Level Sensors
}

\author{
Andrzej Buchacz and Andrzej Wróbel \\ Additional information is available at the end of the chapter \\ http://dx.doi.org/10.5772/54580
}

\section{Introduction}

In recent years there is growing interest of materials, called smart materials. They have one or more properties that can be significantly changed. Smartness describes abilities of shape, size and state of aggregation changes. The main groups of smart materials are:

- piezoelectric plates,

- magneto-rheostatic materials,

- electro-rheostatic materials,

- shape memory alloys.

Those materials are widely used in technology and their numbers of applications still growing. Piezoelectric effect was discovered by French physicists Peter and Paul Curie in the 1880s. They described generation of electric charge on the surface with various shape during its deformation in different directions.

In their research, first of all, they focused on tourmaline crystal, salt and quartz. In 1881s Gabriel Lippman suggested the existence of the reverse piezoelectric phenomenon, which was confirmed experimentally by the Curie brothers. As a solution of research such two, unique properties of piezoelectric materials were assigned:

- showing of simple piezoelectric effect, which rely on generating of voltage after deformation of material,

- reverse piezoelectric effect, which rely on changing of sizes (by around $4 \%$ ) after applying a voltage to piezoelectric facing. 
Designing of technological systems, which contains piezoelectric elements should not be framed only to mechanical system analysis, but should be taken under consideration also electrical part. The entity should be considered as complex system, which contains independent subsystem.

Problem with mechanical-physical systems synthesis, first of all electrical and mechanical ones, is well known and frequently published (Arczewski, 1988; Bellert 1981; Białas, 2012; Buchacz \& Płaczek, 2012). In articles concerned theory and designing of filters not much space was devoted to mechanical systems with parameters distributed in continuous way. Determination tests of mechatronic systems characteristics, applications of graphs and structural numbers were carried out at Silesian Center repeatedly (Buchacz, 2004; Sękala \& Świder, 2005; Wróbel, 2012). Those studies gave assumption to analysis of piezoelectric work. In many publications and papers, mechanical systems investigations on example of vibration beams and rods, were introduced. Moreover, rules of modellinig by non-classical method and attempts of analysis by using hypergraph skeletons (Buchacz \& Świder, 2000), graphs with signal flow and matrix methods (Bishop et at.,1972) were shown in these works.

Nowadays, numerous piezoelectric advantages caused its multi-application in mechanics and in many replaced field of science (Shin et al.,2005; Ha, 2002). Many times beams configurations, with respect to different boundary conditions and during piezoelectric application in damping of vibrations, were analyzed. In the paper (Sherrit, 1999) capability of piezoelectric systems modelling using equivalent Manson models were presented. Analysis of longitudinal vibrations were made taking into consideration dielectric and piezoelement layer. Mason in (Mason, 1948) introduced one-dimensional, equivalent system parameters widely used in modelling systems both free, and loaded. The main disadvantage of such approach is the equivalent of the mechanical system by discreet model. In article (Shin et at.,2005) author presents 4-port equivalent system of piezoelectric plate, used to identification of system response on mechanical force. A matrix, size 5x5, input-output dependences, with different conditions of support, was also determined. Another type of piezoelectric transducer, which was based on Masons alternative systems of higher number of piezoelectric layer, were presented in many articles. Simulation was carried out in frequency domain, furthermore result was compared with values obtained by experimental method. Bellert in his volumes of chosen works (Bellert 1981) many times wrote about modelling of replacing systems, examined as 4-ports. In work (Bolkowski, 1986) author provide chain method of connection electric 4-ports. However, both: (Bolkowski, 1986) and (Bellert 1981) concerned primarily electric systems. In research work number N502 071 31/3719 attempts of active, mechanical systems, with damping in scope of graphs and structural numbers methods, were analyzed. In such, rich publications from field of vibration analysis, solution of piezoelectric plate itself with respect to dynamic characteristic was not undertaken, with the exceptions (Kacprzyk, 1995).

Previous presented solutions were conducted mainly in field of time and concerned single plate. Present paper is continuation of mentioned publications with stack of piezoelectric plates. This work is an author's idea of calculations of complex systems with many elements. The base of calculation is matrix method and application of aggregation of graphs to determination characteristic parameters of bimorphic systems, as well as to drawing its characteristics. 


\section{Vibrating level sensor as a practical example of the application of piezoelectric stacks}

An important characteristic of the designed and analyzed piezoelectric systems is the possibility of their practical application. This chapter presents options for further research related to the piezoelectric phenomenon of complex systems. Both a single piezoelectric plate, as well as complex systems, are often used in pressure, level, force and displacement sensors. As part of future research is proposed execution of laboratory stand for tests of piezoelectric plates used in vibration sensors. These sensors were used for level detection of loose materials in open or pressurized tanks. Output signal is a binary signal, transmitted to the automation systems via a relay. In Fig. 1 and Fig. 2 the level of vibration sensors manufactured by the "Nivomer" company from Gliwice were shown.

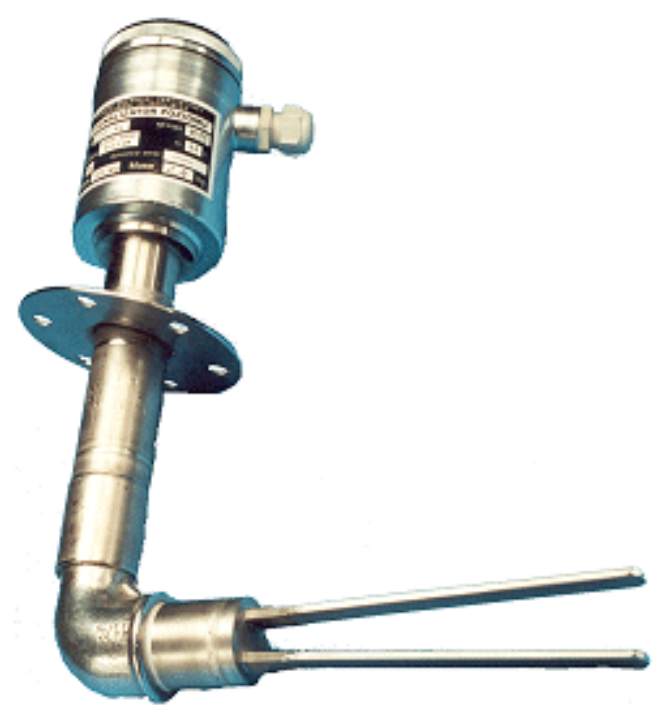

Figure 1. Angular vibrational sensor

The application of stack tiles for intensification of the output signal. The sensors consist of two pairs of receiving plates and two or three supplying plates, connected in a bimorphic system (Fig. 3, Fig. 4). Variable voltage, which feeds the supply plates results in a change in their thickness proportionally to the value of applied voltage.

Changes in the plate thickness causing mechanical vibrations of the element, so called "fork". When the "forks" are not covered by material, full deformation of supplying plates are transferred to the receiving ones. As a result of elongation of receiving plates, on its facing, there is a difference of potentials, proportional to the force. The value of this voltage is 


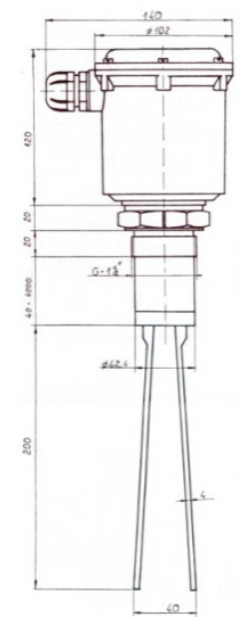

Figure 2. Approximate dimensions [documentation of the "Nivomer"company]

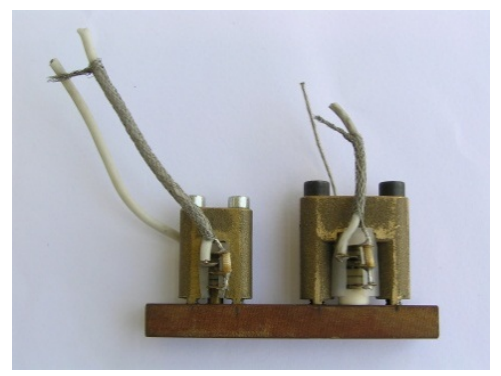

Figure 3. Stack of plates in the level sensor

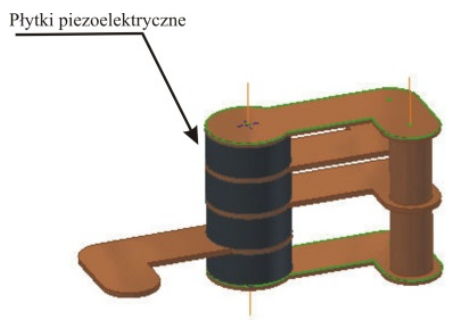

Figure 4. Computer model of the tiles stack

transformed by an electronic system (Fig. 5). In case of covered "forks", the receiving plates are no longer crushed and stretched. At the same time the potential is not generated on the facing of the plates. 


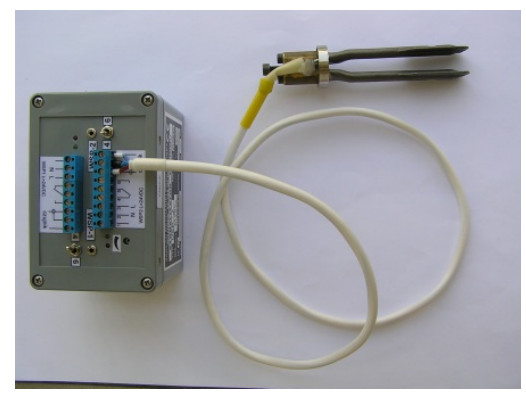

Figure 5. View of the sensor with control

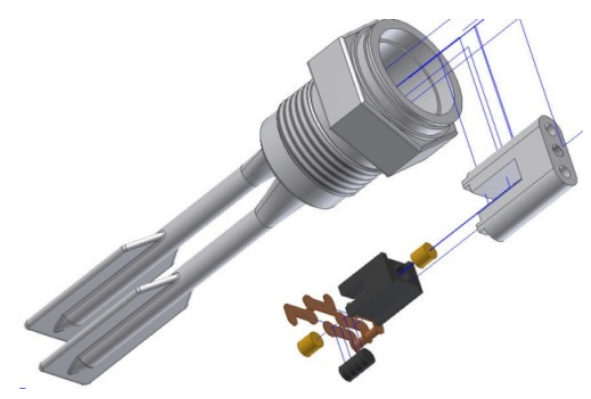

Figure 6. Construction of the level sensor by "Nivomer" company

Described sensor of "Nivomer" company is made of the body, ended with membrane which intensify a signal, to which are welded two identical vibrating rods (Fig. 6). In the presented sensor piezoelectric plates in the form of discs were used but there is a possibility of replacing the plates with shapes analyzed in previous chapters. Effect of plates stacks analysis in sensors designing The proposed analysis of the piezoelectric phenomenon of bimorphic systems allows at the design stage to determine the optimal parameters of piezoelectric plates. Wellchosen plate size and their number is crucial in the performance of other mechanical parts.

After the preliminary analysis of the construction of sensors with the company "Nivomer" from Gliwice found that by introducing a variable number of plates in the system is possible to choose the frequency of the generator and the maximum deflection of the fork carrying vibrations. The proposed methods and algorithms of work concern complex systems, can be used to design a stack of piezoelectric plates in the presented sensor level. In future work, it is proposed to conduct vibration test level sensors and a comparison of the algebraic method with the experimental method. 


\section{Object model under examination}

Under consideration is vibrating piezoelectric plate with parameters distributed in a continuous way. The model has a section $A$, a thickness $d$ and is made of a uniform material with a density $\rho$. Example of such system was shown in Fig. 7. Mechanical displacements are caused by the forces and voltage, while the current is generated by the difference of potentials on the plates of piezoelectric.

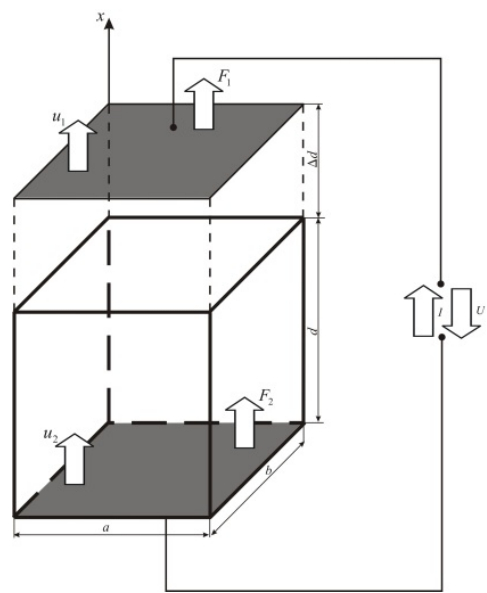

Figure 7. Continuous and limited piezoelectric model

In the analyzed example calculations are based on constitutive equations that include the assumed boundary conditions. In the study assumed that the test object is vibrating piezoelectric plate treated as a one-dimensional system. Piezoelectric plate constitutive equations are as follows (Soluch, 1980):

$$
\left\{\begin{array}{l}
\sigma=E \frac{\partial u}{\partial x}-\varepsilon E_{p^{\prime}} \\
D=\varepsilon^{S} E_{p}+\varepsilon \frac{\partial u}{\partial x}
\end{array}\right.
$$

where:

$E$ - the modulus of longitudinal elasticity,

$E_{P}$ - the intensity value of electric field,

$\varepsilon$ - deformation,

$\varepsilon^{S}$ - the electric permeability, 
$D$ - the electric induction.

The equation of motion of a given element is as follows:

$$
\frac{\partial \sigma}{\partial x}=\rho \ddot{u}
$$

where:

$\sigma$ - tension,

$\rho$ - density of piezoelectric plate.

It was assumed that Poisson equation is:

$$
\frac{\partial D}{\partial x}=0
$$

Rearranging equation (1) due to $E_{p}$ :

$$
E_{p}=\frac{D}{\varepsilon^{S}}-\frac{\varepsilon}{\varepsilon^{S}} \frac{\partial u}{\partial x} .
$$

Substituting expression (4) into equation (1), strain is given:

$$
\sigma=c \frac{\partial u}{\partial x}-\frac{\varepsilon}{\varepsilon^{S}} D
$$

where:

$c=E+\varepsilon^{2} / \varepsilon^{S}$ is the stiffened elastic constant.

From equation (3) result that $D=$ const.Therefore, the equation of motion (2), that takes into account (5), is a one-dimensional wave equation:

$$
c \frac{\partial^{2} u}{\partial x^{2}}=\rho \ddot{u},
$$

or assuming that the volume wave equation in piezoelement is equal to:

$$
V=\sqrt{\frac{c}{\rho}},
$$


equation of motion (6) was presented in the form:

$$
\frac{\partial^{2} u}{\partial x^{2}}-\frac{1}{V^{2}} \ddot{u}
$$

Assuming the expansion of the plate, mainly in the perpendicular plane to the axis, the following boundary conditions were defined:

$$
\left\{\begin{array}{lll}
u=u_{1}, & \text { when } & x=x_{1}, \\
u=u_{2}, & \text { when } & x=x_{2}, \\
\sigma=-\sigma_{1}, & \text { when } & x=x_{1}, \\
\sigma=-\sigma_{2}, & \text { when } & x=x_{2} .
\end{array}\right.
$$

Replaced mechanical stress by force, using the formula $F=A \sigma$, where $A$ is the surface of piezoelectric plate and $\sigma$ is the stress of piezoelectric plate. Determined forces in this case are:

$$
\left\{\begin{array}{l}
F=F_{1}, \text { when } x=x_{1}, \\
F=F_{2}, \text { when } x=x_{2} .
\end{array}\right.
$$

The solution of equation (8) is harmonical displacement:

$$
u=a_{1} e^{j(\omega t-k x)}+a_{1} e^{j(\omega t+k x)}
$$

or assuming, that

$$
A_{1}=a_{1} e^{j \omega t}, A_{2}=a_{2} e^{j \omega t}, A_{2}=a_{2} e^{j \omega t} .
$$

written as:

$$
u=A_{1} e^{-j k x}+A_{2} e^{j k x}
$$

Taking into account the boundary conditions included in the model (9) was obtained:

$$
\left\{\begin{array}{l}
u_{1}=A_{1} e^{-j k x_{1}}+A_{2} e^{j x_{1}}, \\
u_{2}=A_{1} e^{-j k x_{2}}+A_{2} e^{j x_{2}}
\end{array}\right.
$$


In order to determine the coefficients $A_{1}$ and $A_{2}$, presented in equation (12) the determinants method was used. The determinant of the set of equations (14) takes the form:

$$
\begin{gathered}
W=\left|\begin{array}{cc}
e^{-j k x_{1}} & e^{j k x_{1}} \\
e^{-j k x_{2}} & e^{j k x_{2}}
\end{array}\right|, \\
|W|=e^{-j k x_{1}} e^{j k x_{2}}-e^{j k x_{1}} e^{-j k x_{2}}, \\
|W|=e^{-j k x_{1}+j k x_{2}}-e^{-j k x_{2}+j k x_{1}} .
\end{gathered}
$$

Using the dependences between the trigonometric and exponential functions:

$$
\begin{aligned}
& \sin x=\frac{1}{2 j}\left(e^{j x}-e^{-j x}\right), \\
& \cos x=\frac{1}{2}\left(e^{j x}+e^{-j x}\right),
\end{aligned}
$$

equation (17) was written as:

$$
|W|=2 j \sin \left(k\left(x_{2}-x_{1}\right)\right)
$$

and assuming that the thickness of the plate $d=x_{2}-x_{1}$, the equation was determined:

$$
|W|=2 j \sin k d
$$

Determinant $W_{A_{1}}$ results from equation (2.14) and is:

$$
W_{A_{1}}=\left|\begin{array}{ll}
u_{1} & e^{j k x_{1}} \\
u_{2} & e^{j k x_{2}}
\end{array}\right|,
$$

so:

$$
\left|W_{A_{1}}\right|=u_{1} e^{j k x_{2}}-u_{2} e^{j k x_{1}} .
$$


Using the record:

$$
A_{1}=\frac{\left|W_{A}\right|}{|W|}
$$

and substituting the expression (21), (23) into equation (24), $A_{1}$ was obtained:

$$
A_{1}=\frac{u_{1} e^{j k x_{2}}-u_{2} e^{j k x_{1}}}{2 j \sin k d} .
$$

Furthermore, the determinant $W_{A_{2}}$ was calculated:

$$
W_{A_{2}}=\left|\begin{array}{ll}
e^{-j k x_{1}} & u_{1} \\
e^{-j k x_{2}} & u_{2}
\end{array}\right|,
$$

so:

$$
\left|W_{A_{2}}\right|=u_{2} e^{-j k x_{1}}-u_{1} e^{-j k x_{2}},
$$

Analogously to (25) $A_{2}$ was determined:

$$
A_{2}=\frac{u_{2} e^{-j k x_{1}}-u_{1} e^{-j k x_{2}}}{2 j \sin k d} .
$$

Substituting (11) into equation (5) was written:

$$
\sigma=c\left(-j k A_{1} e^{-j k x}+j k A_{2} e^{j k x}\right)-\frac{\varepsilon}{\varepsilon^{S}} D .
$$

Using (10), the force was defined $F_{1}$ :

$$
-F_{1}=-A c k\left(j A_{1} e^{-j k x_{1}}-j A_{2} e^{j k x_{1}}\right)-\frac{\varepsilon}{\varepsilon^{S}} D,
$$

substituting determined $A_{1}, A_{2}$ to the equation (30), was obtained: 


$$
-F_{1}=-A c k\left[j\left(\frac{u_{1} e^{j k x_{2}}-u_{2} e^{j k x_{1}}}{2 j \sin k d}\right) e^{-j k x_{1}}-j\left(\frac{u_{2} e^{-j k x_{1}}-u_{1} e^{-j k x_{2}}}{2 j \sin k d}\right) e^{j k u_{1}}\right]-\frac{\varepsilon}{\varepsilon^{S}} D .
$$

Carrying out the multiplication of expressions in brackets (31):

$$
-F_{1}=-A c k\left[\left(\frac{u_{1} e^{j k\left(x_{2}-x_{1}\right)}-u_{2} e^{j k\left(x_{1}-x_{1}\right)}}{2 \sin k d}\right)-\left(\frac{u_{2} e^{-j k\left(x_{1}-j x_{1}\right)}-u_{1} e^{-j k\left(x_{2}-x_{1}\right)}}{2 \sin k d}\right)\right]-\frac{\varepsilon}{\varepsilon^{S}} D,
$$

and excluding the displacement $u_{1}$ and $u_{2}$ before the bracket:

$$
-F_{1}=-A c k\left[\frac{u_{1}\left(e^{j k\left(x_{2}-x_{1}\right)}+e^{-j k\left(x_{2}-x_{1}\right)}\right)-u_{2}\left(e^{j k\left(x_{1}-x_{1}\right)}+e^{-j k\left(x_{1}-x_{1}\right)}\right)}{2 \sin k d}\right]-\frac{\varepsilon}{\varepsilon^{S}} D
$$

And taking into account the dependence:

$$
\cos k d=\frac{\left(e^{j k\left(x_{2}-x_{1}\right)}+e^{-j k\left(x_{2}-x_{1}\right)}\right)}{2}
$$

was obtained:

$$
-F_{1}=-A c k\left[\frac{u_{1} \cos k d}{\sin k d}-\frac{u_{2}}{\sin k d}\right]-\frac{\varepsilon}{\varepsilon^{S}} D .
$$

Finally, introducing trigonometric functions, equation (35) was written as:

$$
F_{1}=A c k\left[\frac{u_{1}}{\tan k d}-\frac{u_{2}}{\sin k d}\right]+\frac{\varepsilon}{\varepsilon^{S}} D
$$

In (36) the ralationship of force $F_{1}$, acting on the beginning of the system (Fig. 2), on the displacements $u_{1}$ and $u_{2}$ and its influence on the piezoelectric effect $\frac{\varepsilon}{\varepsilon^{S}} D$ were shown. Force $F_{2}$, determined from the equation (30) is equal to:

$$
-F_{2}=-A c k\left(j A_{1} e^{-j k x_{2}}-j A_{2} e^{j k x_{2}}\right)-\frac{\varepsilon}{\varepsilon^{S}} D,
$$


and substituting the determinants $A_{1}(25), A_{2}(28)$ to the equation (30), was obtained:

$$
-F_{2}=-A c k\left[j\left(\frac{u_{1} e^{j k x_{2}}-u_{2} e^{j k x_{1}}}{2 j \sin k d}\right) e^{-j k x_{2}}-j\left(\frac{u_{2} e^{-j k x_{1}}-u_{1} e^{-j k x_{2}}}{2 j \sin k d}\right) e^{j k u_{2}}\right]-\frac{\varepsilon}{\varepsilon^{S}} D .
$$

Carrying out the multiplication of expressions in brackets:

$$
-F_{2}=-A c k\left[\left(\frac{u_{1} e^{j k\left(x_{2}-x_{2}\right)}-u_{2} e^{-j k\left(x_{2}-x_{1}\right)}}{2 \sin k d}\right)-\left(\frac{u_{2} e^{j k\left(x_{2}-j x_{1}\right)}-u_{1} e^{-j k\left(x_{2}-x_{2}\right)}}{2 \sin k d}\right)\right]-\frac{\varepsilon}{\varepsilon^{S}} D,
$$

and excluding displacements $u_{1} \mathrm{I} u_{2}$ before brackets:

$$
-F_{2}=-A c k\left[\frac{u_{1}\left(e^{j k\left(x_{2}-x_{2}\right)}+e^{-j k\left(x_{2}-x_{2}\right)}\right)-u_{2}\left(e^{-j k\left(x_{2}-x_{1}\right)}+e^{j k\left(x_{2}-x_{1}\right)}\right)}{2 \sin k d}\right]-\frac{\varepsilon}{\varepsilon^{S}} D,
$$

and using dependences $(18,19)$ written:

$$
-F_{2}=-A c k\left[\frac{u_{1}}{\sin k d}-\frac{u_{2} \cos k d}{\sin k d}\right]-\frac{\varepsilon}{\varepsilon^{S}} D .
$$

Finally, taking into account the trigonometric functions,

$$
F_{2}=A c k\left[\frac{u_{1}}{\sin k d}-\frac{u_{2}}{\tan k d}\right]+\frac{\varepsilon}{\varepsilon^{S}} D
$$

was calculated.

Dependences of the forces and displacements acting on the system under consideration, taking into account the piezoelectric effect, are taking the following form:

$$
\left\{\begin{array}{l}
F_{1}=\rho V A\left(\frac{u_{1}}{\tan k d}-\frac{u_{2}}{\sin k d}\right)+\frac{\varepsilon}{\varepsilon^{S}} D, \\
F_{2}=\rho V A\left(\frac{u_{1}}{\sin k d}-\frac{u_{2}}{\tan k d}\right)+\frac{\varepsilon}{\varepsilon^{S}} D .
\end{array}\right.
$$


Analyzing the effects occurring in the piezoelectric plate, also the electrical parameters such as voltage on the plates of piezoelectric and current value were took into account. Voltage $U$ is therefore expressed as a function of electric field:

$$
U=\int_{u 1}^{u 2} E_{p} d u,
$$

where:

$U$ - value of generated voltage on the linings of piezoelectric,

$E_{p}$ - electric field intensity.

Integrating (44):

$$
U=\frac{D d}{\varepsilon^{s}}-\frac{\varepsilon}{\varepsilon^{s}}\left(u_{2}-u_{1}\right)
$$

where:

$D$ - electric induction module, defined as:

$$
D=\frac{i}{\omega A}
$$

finally the voltage in a function of current was shown as:

$$
U=\frac{h}{\omega}\left(u_{2}-u_{1}\right)+\frac{1}{\omega C_{0}} i
$$

where:

$$
h=\frac{\varepsilon}{\varepsilon^{s}} .
$$

Capacitance, depends directly on the dimensions of the plates, aswell as physicochemical properties, written in the form:

$$
C_{0}=\frac{\varepsilon^{s} A}{d}
$$


Introducing (46) into equations (36), (42), (46), dependences of replacement set of piezoelectric plate were received, plate characterized by three equations:

$$
\begin{gathered}
F_{1}=A c k\left[\frac{u_{1}}{\tan k d}-\frac{u_{2}}{\sin k d}\right]+\frac{\varepsilon}{\varepsilon^{S}} \frac{i}{\omega A}, \\
F_{2}=A c k\left[\frac{u_{1}}{\tan k d}-\frac{u_{2}}{\sin k d}\right]+\frac{\varepsilon}{\varepsilon^{S}} \frac{i}{\omega A}, \\
U=\frac{h}{\omega}\left(u_{2}-u_{1}\right)+\frac{1}{\omega C_{0}} i .
\end{gathered}
$$

Assuming signs :

$$
k=\frac{\omega}{V}
$$

and

$$
Z=\rho V A,
$$

obtained the relations between the electrical and mechanical values of piezoelectric plates:

$$
\begin{gathered}
F_{1}=Z\left[\frac{u_{1}}{\tan k d}-\frac{u_{2}}{\sin k d}\right]+\frac{h i}{\omega}, \\
F_{2}=Z\left[\frac{u_{1}}{\tan k d}-\frac{u_{2}}{\sin k d}\right]+\frac{h i}{\omega}, \\
U=\frac{h}{\omega}\left(u_{2}-u_{1}\right)+\frac{1}{\omega C_{0}} i
\end{gathered}
$$

which are also written in a matrix form: 


$$
\left[\begin{array}{c}
F_{1} \\
U \\
F_{2}
\end{array}\right]=\left[\begin{array}{ccc}
\frac{Z}{\tan k d} & \frac{h}{\omega} & -\frac{Z}{\sin k d} \\
\frac{h}{\omega} & \frac{1}{\omega C_{0}} & -\frac{h}{\omega} \\
\frac{Z}{\sin k d} & \frac{h}{\omega} & -\frac{Z}{\tan k d}
\end{array}\right]\left[\begin{array}{c}
u_{1} \\
i \\
u_{2}
\end{array}\right] .
$$

\section{Mapping matrix into graph}

The values of susceptibility, admittance and characteristics were determined from the formula:

$$
Y=\frac{1}{\operatorname{det} Z}\left(\left(Z^{M}\right)^{D}\right)^{T}
$$

The matrix (58) was written as respond of system for operating extortion. The individual elements of matrix, presented as a flexibility, admittance and characteristics were recorded as follows: determined dependences of force from displacement are given in Table 1.

\begin{tabular}{|c|c|c|c|}
\hline & $F_{1}$ & $U$ & $F_{2}$ \\
\hline$u_{1}$ & $\frac{\left(\frac{h^{2}}{\omega^{2}}\right)-\left(\frac{\rho V A}{\omega C_{0} \tan k d}\right)}{\operatorname{det} Z}$ & - & $\frac{-\left(\frac{h^{2}}{\omega^{2}}\right)+\left(\frac{1}{\omega C_{0}} \frac{\rho V A}{\sin k d}\right)}{\operatorname{det} Z}$ \\
\hline$i$ & - & - & - \\
\hline$u_{2}$ & $\frac{\left(\frac{h^{2}}{\omega^{2}}\right)-\left(\frac{1}{\omega C_{0}} \frac{\rho V A}{\sin k d}\right)}{\operatorname{det} Z}$ & - & $\frac{-\left(\frac{h^{2}}{\omega^{2}}\right)+\left(\frac{\rho V A}{\omega C_{0} \tan k d}\right)}{\operatorname{det} Z}$ \\
\hline
\end{tabular}

Table 1. Dependences of force from displacement

The dependences between mechanical and electrical parameters were shown in Table 2.

In table 3 electrical dependences: voltage and current, so called admittance of piezoelectric system, were listed.

In order to determine graph, representing modeled system of piezoelectric plates, as the symbols in the matrix were used. The elements of matrix (58) assigned to the edges of graph are presented as: 


\begin{tabular}{|c|c|c|c|}
\hline & $F_{1}$ & $U$ & $F_{2}$ \\
\hline$u_{1}$ & - & $\frac{\frac{h \rho V A}{\omega}\left(\frac{1}{\tan k d}-\frac{1}{\sin k d}\right)}{\operatorname{det} Z}$ & - \\
\hline$i$ & $\frac{\frac{h \rho V A}{\omega}\left(\frac{1}{\tan k d}-\frac{1}{\sin k d}\right)}{\operatorname{det} Z}$ & - & $\frac{h \rho V A}{\omega}\left(\frac{1}{\tan k d}-\frac{1}{\sin k d}\right)$ \\
\hline$u_{2}$ & - & $\frac{-\frac{h \rho V A}{\omega}\left(\frac{1}{\tan k d}+\frac{1}{\sin k d}\right)}{\operatorname{det} Z}$ & - \\
\hline
\end{tabular}

Table 2. Dependences between mechanical and electrical parameters

\begin{tabular}{|c|c|c|c|}
\hline & $F_{1}$ & $U$ & $F_{2}$ \\
\hline$u_{1}$ & - & - & - \\
$i$ & - & $-\left(\frac{\rho V A}{\tan k d}\right)^{2}+\left(\frac{\rho V A}{\sin k d}\right)^{2}$ & - \\
\hline$u_{2}$ & - & $\operatorname{det} Z$ & - \\
\hline
\end{tabular}

Table 3. Electrical dependences: voltage and current

$$
\begin{gathered}
Y_{11}=\left(x_{1}, F_{1}\right)=\frac{1}{\operatorname{det} Z}\left\{-\frac{\rho V A}{\omega C_{0} \operatorname{tg} k d}-\frac{h^{2}}{\omega^{2}}\right\} \\
Y_{12}=\left(x_{1}, U\right)=\frac{1}{\operatorname{det} Z}\left\{-\frac{\rho V A}{\sin k d} \frac{h}{\omega}-\frac{h}{\omega} \frac{\rho V A}{\sin k d}\right\} \\
Y_{13}=\left(x_{1}, F_{2}\right)=\frac{1}{\operatorname{det} Z}\left\{\frac{h^{2}}{\omega^{2}}-\frac{1}{\omega C_{0}} \frac{\rho V A}{\sin k d}\right\}
\end{gathered}
$$




$$
\begin{gathered}
Y_{21}=\left(i, F_{1}\right)=\frac{1}{\operatorname{det} Z}\left\{\frac{h}{\omega} \frac{\rho V A}{\operatorname{tg} k d}+\frac{\rho V A}{\sin k d} \frac{h}{\omega}\right\} \\
Y_{22}=(i, U)=\frac{1}{\operatorname{det} Z}\left\{-\left(\frac{\rho V A}{\operatorname{tg} k d}\right)^{2}+\left(\frac{\rho V A}{\sin k d}\right)^{2}\right\} \\
Y_{23}=\left(i, F_{2}\right)=\frac{1}{\operatorname{det} Z}\left\{-\frac{\rho V A}{\omega C_{0} \operatorname{tg} k d}-\frac{h^{2}}{\omega^{2}}\right\} \\
Y_{31}=\left(x_{2}, F_{1}\right)=\frac{1}{\operatorname{det} Z}\left\{\frac{h^{2}}{\omega^{2}}+\frac{\rho V A}{\omega C_{0} \sin k d}\right\} \\
Y_{32}=\left(x_{2}, U\right)=\frac{1}{\operatorname{det} Z}\left\{\frac{h}{\omega} \frac{\rho V A}{\operatorname{tg} k d}-\frac{\rho V A}{\sin k d} \frac{h}{\omega}\right\} \\
Y_{33}=\left(x_{2}, F_{2}\right)=\frac{1}{\operatorname{det} Z}\left\{\frac{h^{2}}{\omega^{2}} \frac{\rho V A}{\operatorname{tg} k d}-\frac{\rho V A}{\omega C_{0} \operatorname{tg} k d}\right\}
\end{gathered}
$$

The graphical representation of mapping is shown as:

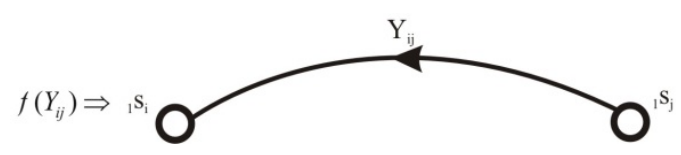

Figure 8. Mapping $Y_{i j}$

The symbol $Y_{i j}$ is the mechanical flexibility, electrical admittance or characteristics of the system. In mapping of the parameters into the graph, mark $Y_{i j}$ means the relationship between the vertex of graph, directed from the apex $i$ to apex $j$, with the symbol $i=j$, then the following relationship were true:

$Y_{11}=Y_{10}$

$$
Y_{22}=Y_{20}
$$

$Y_{33}=Y_{30}$ 
Dependences according to the index $j=0$ maps a connection of the vertex with the base vertex. Following this systematic, assignment by an edge of following relations was made:

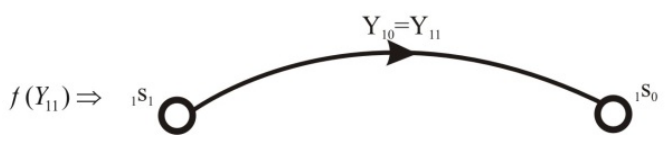

Figure 9. Mapping $Y_{10}$

where $f\left(Y_{11}\right)$ is the mechanical flexibility;

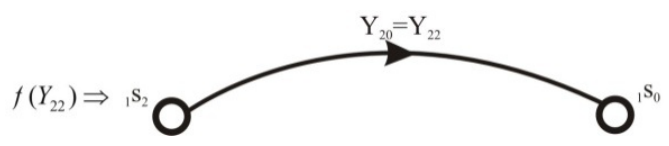

Figure 10. Mapping $Y_{20}$

where $f\left(Y_{22}\right)$ is admittance of electrical system;

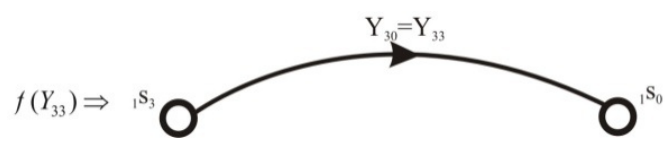

Figure 11. Mapping $Y_{30}$

where $f\left(Y_{33}\right)$ is mechanical flexibility;

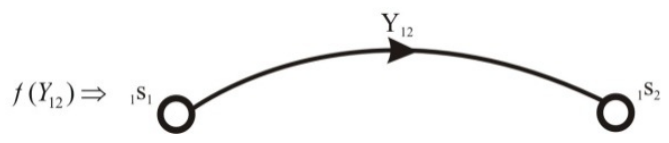

Figure 12. Mapping $Y_{12}$

where $f\left(Y_{12}\right)$ is system characteristic;

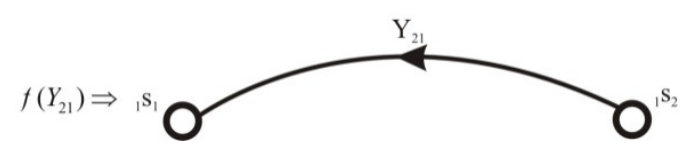

Figure 13. Mapping $Y_{21}$ 
where $f\left(Y_{21}\right)$ is system characteristic;

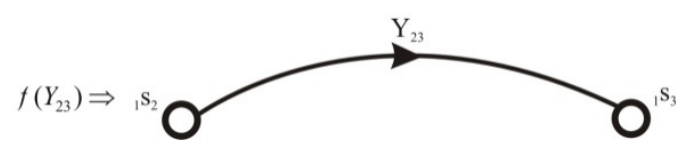

Figure 14. Mapping $Y_{23}$

where $f\left(Y_{23}\right)$ is system characteristic;

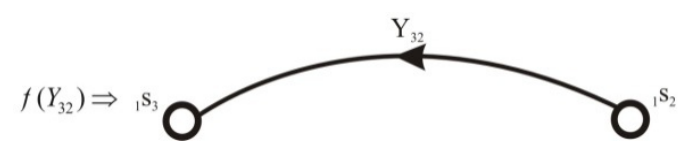

Figure 15. Mapping $Y_{32}$

where $f\left(Y_{32}\right)$ is mechanical flexibility;

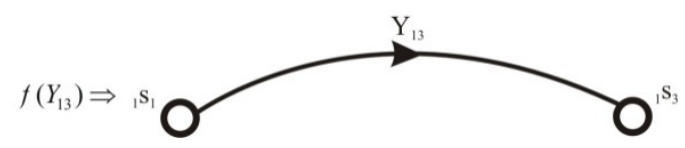

Figure 16. Mapping $Y_{13}$

where $f\left(Y_{13}\right)$ is mechanical flexibility;

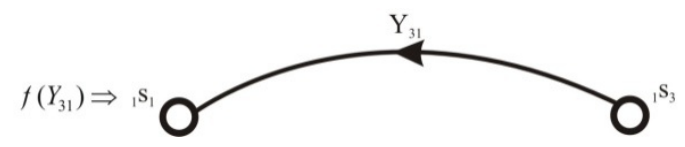

Figure 17. Mapping $Y_{31}$

where $f\left(Y_{31}\right)$ is mechanical flexibility.

A set of drawings of the relation (fig. 9.) - (fig.17.), represents 4-vertex graph, were created and presented in Fig. 18.

$$
{ }_{2} X=\left\{Y_{11}, Y_{22}, Y_{33}, Y_{12}, Y_{21}, Y_{31}, Y_{13}, Y_{23}, Y_{32}\right\}
$$




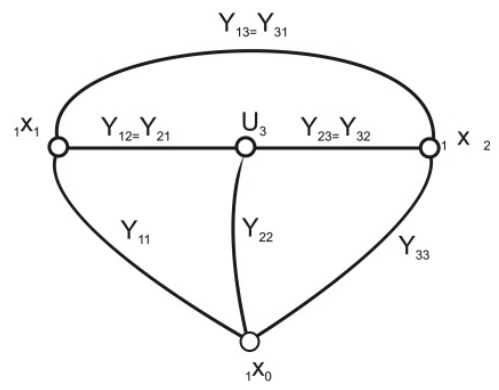

Figure 18. Geometric representation of mapping in the graph

In the rest of the work earlier created 4-vertex graph was replaced by structural number method to the 3-vertex graph.

\section{Construction of the replacement graph}

Furthermore, the use of an extended 4-vertex graph may prove to complicated calculations. In such case, a modelling of system using the replaced graph was performed. In order to maintain clearness of mapping, characteristics determined in paragraph 4 are indicated by Arabic numerals in parentheses, in accordance with (Bellert, 1981). As a consequence of introduction of the replaced graph, a graph presented in Fig. 19 was obtained. It is the basis for further network analysis methods.
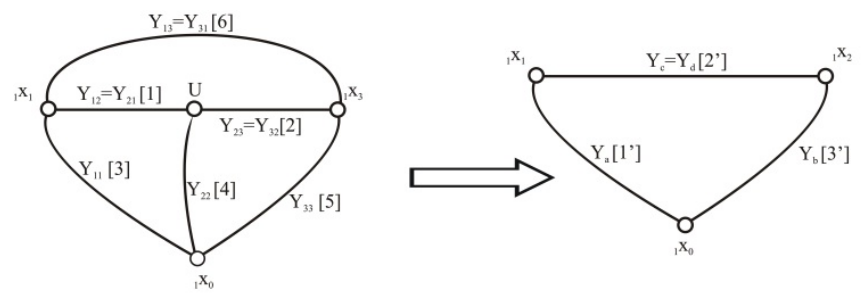

Figure 19. Construction of the replacement graph

As a result of insertion of replaced graph, replaced flexibility of the system was calculated by structural number method:

$$
Y_{b}=Y_{3^{\prime}}=\frac{\operatorname{det}\left(A_{1^{\prime}} \cap A_{2^{\prime}}\right)}{\operatorname{det} A_{1^{\prime} 2^{\prime}}}=\frac{Y_{4}\left(Y_{2}+Y_{5}\right)+Y_{5}\left(Y_{1}+Y_{3}\right)}{Y_{1}+Y_{2}+Y_{4}}
$$




$$
Y_{a}=Y_{1}=\frac{\operatorname{det}\left(A_{2^{\prime}} \cap A_{3^{\prime}}\right)}{\operatorname{det}_{Z} A_{2^{\prime} 3^{\prime}}}=\frac{Y_{1}\left(Y_{3}+Y_{4}\right)+Y_{3}\left(Y_{2}+Y_{4}\right)}{Y_{1}+Y_{2}+Y_{4}}
$$

\section{Chain equation of simple and complex system plate}

On the figure 14 a piezoelectric plate with parameters distributed in the continuous way, the left and right end is free, was presented. The model of a single plate was marked by (i). Currently considered a model system is reduced system in the previous graph from 4-vertex to 3-vertex graph, as shown in Fig. 20.

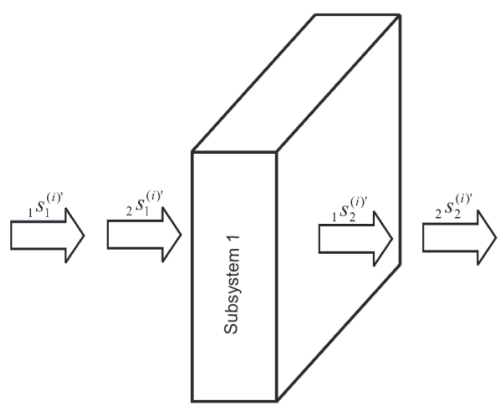

Figure 20. Model of single piezoelectric plate after reduction

Longitudinal vibrations of piezoelectric plate were considered, in the literature described also as thickness. The parameters specifying the system, in accordance with the previously accepted assumptions, were the sizes of input ${ }_{1} s_{1^{\prime}{ }^{2} S_{1}}$ and output ${ }_{1} s_{2^{\prime}{ }^{\prime} S_{2}}$ values, which were presented as:

$$
{ }_{1} S^{(i)}=Y_{2} S^{(i)}
$$

where:

$Y$ is a value characterized input-output dependences.

The relations between displacements of plate, and the forces acting on them, written in matrix form:

$$
\left[\begin{array}{c}
{ }_{1} s_{1}^{(i)} \\
{ }_{1} s_{2}^{(i)}
\end{array}\right]=\left[\begin{array}{cc}
Y_{a}^{(i)} & Y_{c}^{(i)} \\
Y_{d}^{(i)} & Y_{b}^{(i)}
\end{array}\right]\left[\begin{array}{c}
{ }_{2} s_{2}^{(i)} \\
{ }_{2} s_{2}^{(i)}
\end{array}\right]
$$

Transforming the matrix (26) to the chain form expects to receive in the form of matrices: 


$$
\left[\begin{array}{c}
{ }_{2} s_{1}^{(i)} \\
{ }_{1} s_{1}^{(i)}
\end{array}\right]=\left[\begin{array}{ll}
A_{11}^{(i)} & A_{12}^{(i)} \\
A_{21}^{(i)} & A_{22}^{(i)}
\end{array}\right]\left[\begin{array}{c}
{ }_{2} s_{2}^{(i)} \\
{ }_{1} s_{2}^{(i)}
\end{array}\right]
$$

where:

$$
\left\{\begin{array}{l}
A_{11}^{(i)}=\frac{Y_{b}^{(i)}}{Y_{c}^{(i)}}, \\
A_{12}^{(i)}=\frac{1}{Y_{c}^{(i)}}, \\
A_{21}^{(i)}=\frac{Y_{c}^{(i)} Y_{d}^{(i)}-Y_{a}^{(i)} Y_{b}^{(i)}}{Y_{d}^{(i)}} \\
A_{22}^{(i)}=\frac{Y_{a}^{(i)}}{Y_{d}^{(i)}} .
\end{array}\right.
$$

In Fig. 21 the free system, consisting of two plates was presented. Superscript indicates the subsequent number of subsystem.

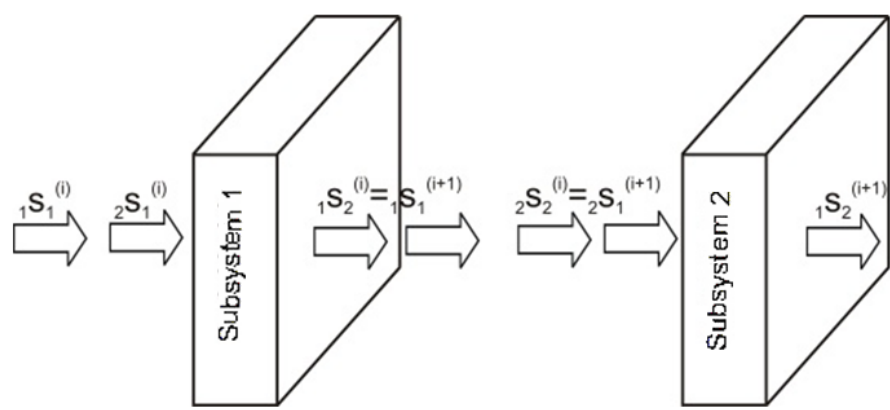

Figure 21. Diagram of a connection between the two cells and the relation between them

$$
B=\left[\begin{array}{l}
{ }_{2} s_{1}^{(i)} \\
{ }_{1} s_{1}^{(i)}
\end{array}\right]=\left[\begin{array}{ll}
A_{11}^{(i)} & A_{12}^{(i)} \\
A_{21}^{(i)} & A_{22}^{(i)}
\end{array}\right]\left[\begin{array}{ll}
A_{11}^{(i+1)} & A_{12}^{(i+1)} \\
A_{21}^{(i+1)} & A_{22}^{(i+1)}
\end{array}\right]\left[\begin{array}{l}
{ }_{2} s_{2}^{(i+1)} \\
{ }_{1} s_{2}^{(i+1)}
\end{array}\right]
$$

Finally, chain equation was written in general form:

$$
A^{(k)}=A^{(i)} A^{(i+1)} .
$$


After the operations carried out according to (79) it was found, that the chain matrix with cascade structure is the ratio of chain matrix of individual cells of the complex system. Obtained transition matrix is presented as:

$$
B=\left[\begin{array}{c}
{ }_{2} s_{1}^{(i)} \\
{ }_{1} s_{1}^{(i)}
\end{array}\right]=\left[\begin{array}{cc}
A_{11}^{(i+1)} A_{11}^{(i)}+A_{12}^{(i)} A_{21}^{(i+1)} & A_{11}^{(i)} A_{12}^{(i+1)}+A_{12}^{(i)} A_{22}^{(i+1)} \\
A_{21}^{(i)} A_{11}^{(i+1)}+A_{22}^{(i)} A_{21}^{(i+1)} & A_{21}^{(i)} A_{12}^{(i)}+A_{22}^{(i)} A_{22}^{(i+1)}
\end{array}\right]\left[\begin{array}{c}
{ }_{2} s_{2}^{(i+1)} \\
{ }_{1} s_{2}^{(i+1)}
\end{array}\right]
$$

Calculated coefficients (80) were substituted and the final form of the transition matrix was received:

$$
\begin{gathered}
B=\left[\begin{array}{ll}
B_{11}^{(k)} & B_{12}^{(k)} \\
B_{21}^{(k)} & B_{22}^{(k)}
\end{array}\right] \\
\left\{\begin{array}{l}
B_{11}^{(k)}=\frac{Y_{b}^{(i)} Y_{b}^{(i+1)}}{Y_{d}^{(i)} Y_{d}^{(i+1)}}+\frac{-Y_{a}^{(i+1)} Y_{b}^{(i+1)}+Y_{c}^{(i+1)} Y_{d}^{(i+1)}}{Y_{d}^{(i)} Y_{d}^{(i+1)}} \\
B_{12}^{(k)}=\frac{Y_{b}^{(i)}}{Y_{d}^{(i)} Y_{d}^{(i+1)}}+\frac{Y_{a}^{(i+1)}}{Y_{c}^{(i)} Y_{d}^{(i+1)}}, \\
B_{21}^{(k)}=\frac{\left(-Y_{a}^{(i)} Y_{b}^{(i)}+Y_{c}^{(i)} Y_{d}^{(i)}\right) Y_{b}^{(i+1)}}{Y_{d}^{(i)} Y_{d}^{(i+1)}}+\frac{Y_{a}^{(i)}\left(-Y_{a}^{(i+1)} Y_{b}^{(i+1)}+Y_{c}^{(i+1)} Y_{d}^{(i+1)}\right)}{Y_{d}^{(i)} Y_{d}^{(i+1)}} \\
B_{22}^{(k)}=\frac{-Y_{a}^{(i)} Y_{b}^{(i)}+Y_{c}^{(i)} Y_{d}^{(i)}}{Y_{d}^{(i)} Y_{d}^{(i+1)}}+\frac{Y_{a}^{(i)} Y_{a}^{(i+1)}}{Y_{d}^{(i)} Y_{d}^{(i+1)}},
\end{array}\right.
\end{gathered}
$$

In order to obtain the flexibility of the complex system, calculated coefficients of chain equation (79), was transformed to the basic form:

$$
\left\{\begin{array}{l}
Y_{a}^{(k)}=\frac{B_{22}{ }^{(k)}}{B_{12}{ }^{(k)}} \\
Y_{c}^{(k)}=-\frac{B_{11}{ }^{(k)} B_{22}{ }^{(k)}}{B_{12}{ }^{(k)}}-B_{21}{ }^{(k)}, \\
Y_{d}^{(k)}=\frac{1}{B_{12}{ }^{(k)}} \\
Y_{b}^{(k)}=\frac{B_{11}{ }^{(k)}}{B_{12}{ }^{(k)}} .
\end{array}\right.
$$


Equation (83) is the components of the complex characteristics of the matrix taking into account obtained chain parameters of complex system.

\section{Charts of simple and bimorph system}

In this paragraph, graphical charts of characteristics of piezoelectric plates were shown. The parameters adopted for graphs plotting was presented in table 4.

\begin{tabular}{llcl}
\hline No. & Symbol & Value & Unit \\
\hline 1 & $\rho$ & 7.5 & {$\left[\frac{g}{\mathrm{~cm}^{3}}\right]$} \\
\hline 2 & $E=c_{33}$ & 150 & {$[\mathrm{GPa}]$} \\
\hline 3 & $A$ & 3.1 & {$\left[\mathrm{~cm}^{2}\right]$} \\
\hline 4 & $d$ & 1 & {$[\mathrm{~mm}]$} \\
\hline
\end{tabular}

Table 4. The parameters adopted for graphs plotting

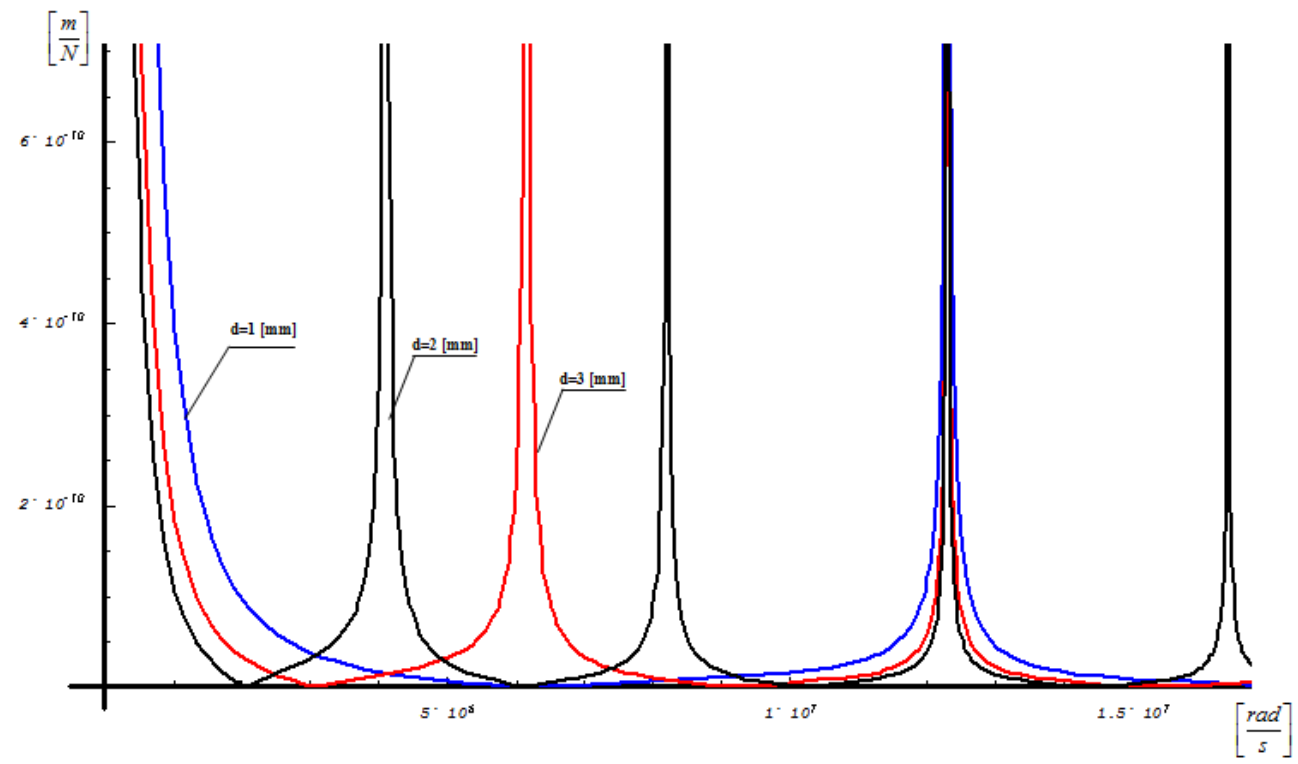

Figure 22. Characteristics of a single piezoelectric plate in the frequency domain, depending on the thickness of the plate 


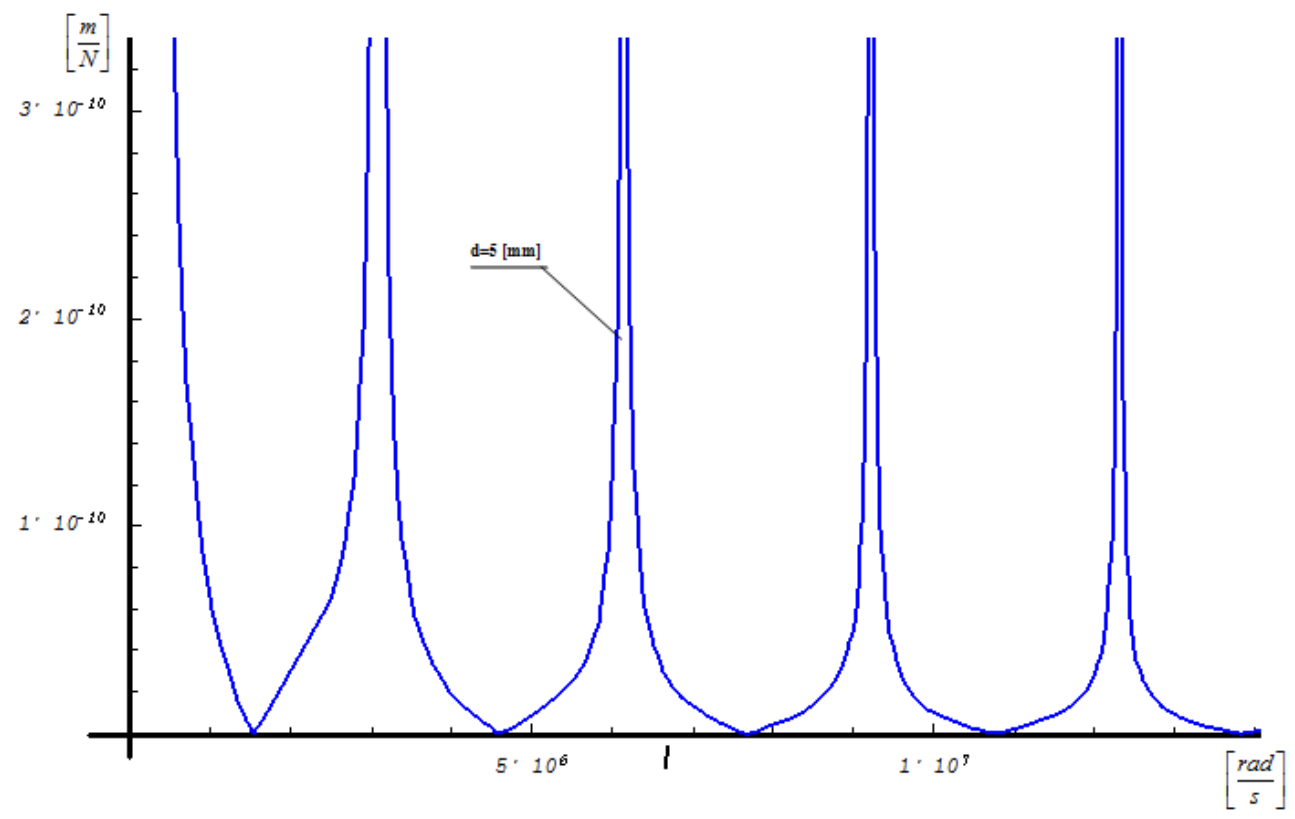

Figure 23. Characteristics of the combined plates of thickness 2 and $3[\mathrm{~mm}]$ in frequency domain

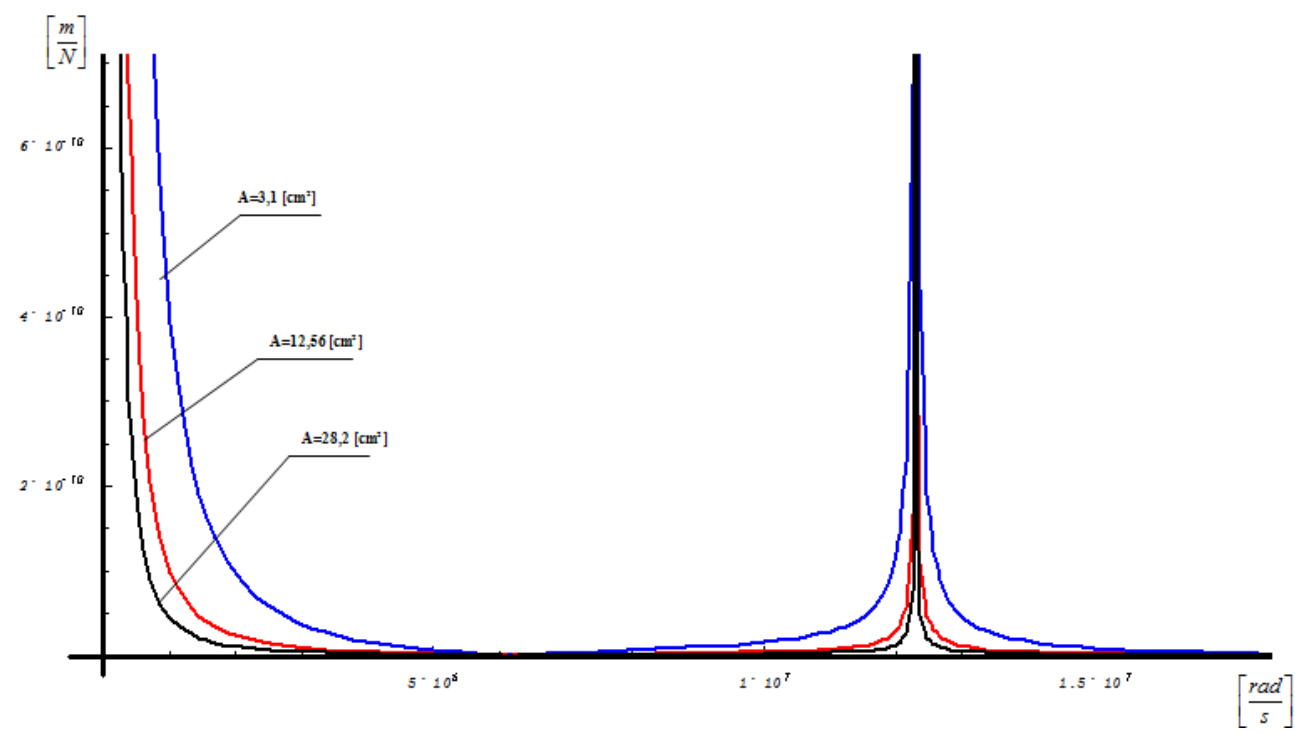

Figure 24. Characteristics of a single piezoelectric plate in the frequency domain, depending on the plate surface area 


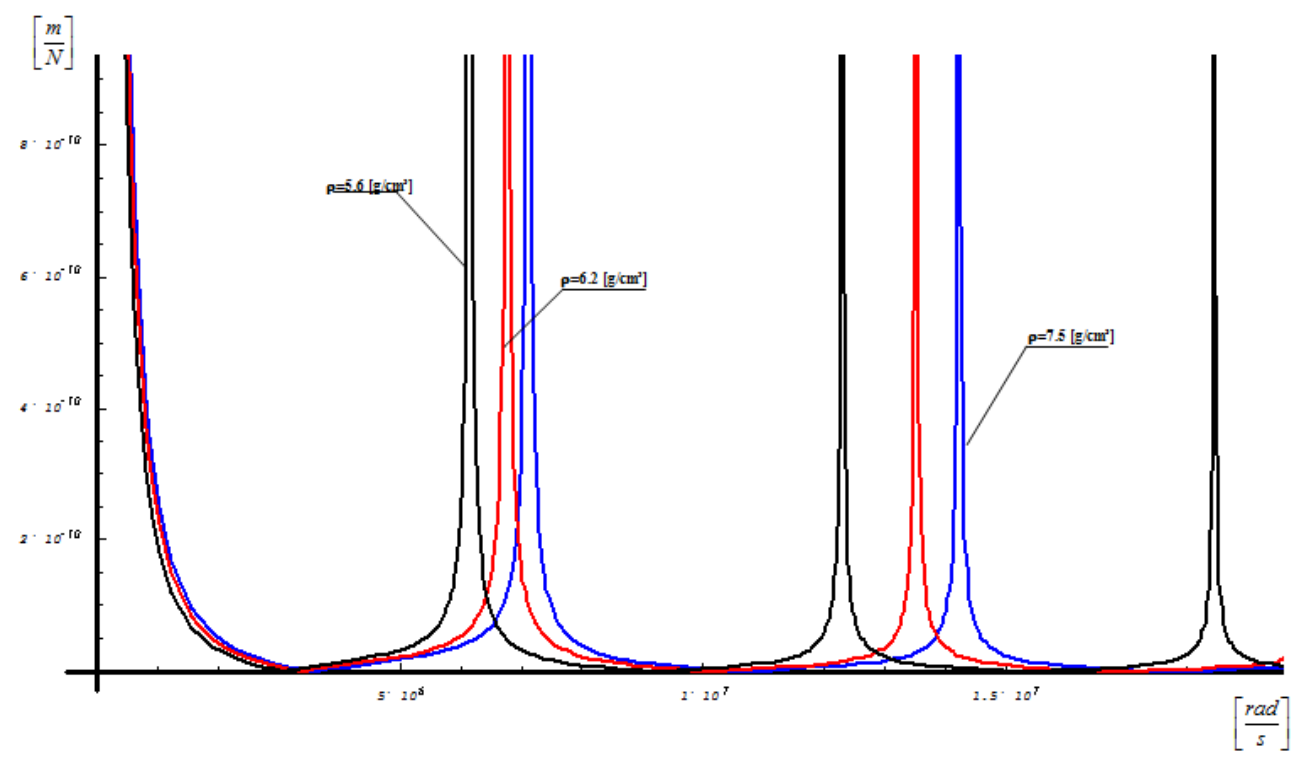

Figure 25. Characteristics of a single piezoelectric plate in the frequency domain, depending on the piezoelectric density

\section{Conclusions}

The chapter concerns the analysis of simple and complex piezoelectric systems, in order to determine the impact of piezoelectric plates parameters on the characteristics of the system. For a long time in the machine building are used subassemblies, whose operation is based on the piezoelectric phenomenon. In a researches of machine elements, on their surface piezoelectric sensors are glued, whereas to monitor the state plates are used transducers made from piezoelectric foil. Piezoelectric are often used in machine building also as assemblies, subassemblies or executive elements. Implementation of the piezoelectric system, which acts as a sensor or actuator is based on the selection of geometric dimensions of the plate, and their basic material parameters. In systems composed of several layers is also important piezoelectric plate number. Moreover, there are new problems at the design stage for the designers working in the field of machine building, concerning the application of both: single and stack plates. This matter is extremely important in terms of practical applications. For this reason it is necessary to conduct research whose main objective is to understand the phenomena associated with vibrations of complex piezoelectric systems.

Work is a continuation and development of decades researches at the Gliwice Center, consist in making the analysis of both the classical methods and non-classical. Take advantage of nonclassical methods is a more general proposes from modeling in classical meaning. Resolves 
simple and complex systems irrespective of the type and number of elements included in the test system.

Applied method of structural numbers method was presented and used previously in modeling mechanical systems.

\section{Author details}

Andrzej Buchacz and Andrzej Wróbel

Silesian University of Technology, Poland

\section{References}

[1] Arczewski, K. (1988). Structural Methods of the Complex Mechanical Systems Analysis, WPW, Warsaw

[2] Behrens, S, Fleming, A. J, \& Moheimani, S. O. R. (2003). Abroadband controller for shunt piezoelectric damping of structural vibration. Smart Materials and Structures, , 12, 18-28.

[3] Bellert, S. (1981). Chosen works, PWN, 8-30100-247-6

[4] Bialas, K. (2012). Mechanical and electrical elements in reduction of vibrations, Journal of vibroengineering, 1392-8716, 14(1), 123-128.

[5] Bishop, R. E. D, Gladwell, G. M. L, \& Michaelson, S. (1972). Matrix analysis of vibration. WNT, Warsaw

[6] Bolkowski, S. (1986). Theoretical electrical engineering. WNT, Warsaw

[7] Buchacz, A. (2004). Hypergrphs and their subgraphs in modelling and investigation of robots. Journal of materials processing technology. Complete, Elsevier, , 157-158, 3744.

[8] Buchacz, A, \& Placzek, M. (2012). The analysis of a composite beam with piezoelectric actuator based on the approximate method, Journal of vibroengineering, 1392-8716, 14(1), 111-116.

[9] Buchacz, A, \& Swider, J. (2000). Skeletons hypergraph in modeling, examination and position robot's manipulator and subassembly of machines. Silesian University of Technology Press, Gliwice.

[10] Ha, S. K. (2002). Analysis of a piezoelectric multimorph in extensional and flexular motions. Journal of Sound and Vibration, 253, 3, , 1001-1014.

[11] Kacprzyk, R, Motyl, E, Gajewski, J. B, \& Pasternak, A. (1995). Piezoelectric properties of nouniform electrets, Journal of Electrostatics 35, , 161-166. 
[12] Mason, W. P. (1948). Electromechanical Transducers and Wale Filters. Van Nostrand

[13] Sekala, A, \& Swider, J. (2005). Hybrid Graphs in Modelling and Analysis of Discrete-Continuous Mechanical Systems. Journal of Materials Processing Technology, Complete Elsevier , 164-165, 1436-1443.

[14] Sherrit, S, Leary, S. P, \& Dolgin, B. P. (1999). Comparison of the Mason and KLM equivalent circuits for piezoelectric resonators in thickness mode, Ultrasonics Symposium.

[15] Shin, H, Ahn, H, \& Han, D. Y. (2005). Modeling and analysis of multilayer piezoelectric transformer, Materials chemistry and physics , 92, 616-620.

[16] Soluch, W. (1980). The introduction to piezoelectronics, WKiE, 8-32060-041-3

[17] Wróbel, A. (2012). Model of piezoelectric including material damping, Proceedings of 16th International Conference ModTech 2012, 2069-6736, 1061-1064. 